Orthopäde $2016 \cdot 45: 925$

DOI 10.1007/s00132-016-3322-1

Online publiziert:22. August 2016

๑) Springer-Verlag Berlin Heidelberg 2016

CrossMark

\author{
A. Eisenschenk ${ }^{1,2}$ \\ ${ }^{1}$ Abteilung für Hand-, Replantations- und Mikrochirurgie, BG Klinikum Unfallkrankenhaus Berlin gGmbH, \\ Berlin, Deutschland \\ ${ }^{2}$ Dieter Buck-Gramcko-Stiftungsprofessur, Hand-und funktionelle Mikrochirurgie, Universitätsmedizin \\ Greifswald, Greifswald, Deutschland
}

\title{
Das Skaphoid
}

Das Os scaphoideum ist der am weitesten radial gelegene Handwurzelknochen der proximalen Reihe. Er artikuliert mit dem Radius, dem Os lunatum, Os capitatum, Os trapezium und Os trapezoideum und ist letztendlich von zentraler Bedeutung für die Beweglichkeit und Biomechanik der Hand. Statistisch stellen die Skaphoidfrakturen ca. $2 \%$ aller Knochenfrakturen, ca. $11 \%$ der Handfrakturen und $60 \%$ der Frakturen der Handwurzelknochen dar. Neben dieser sehr wesentlichen Bedeutung gibt die Blutversorgung des Skaphoids immer wieder Anlass zu Diskussionen. Aufgrund neuerer Untersuchungen kann davon ausgegangen werden, dass der proximale Pol eine ausreichend gute Kollateralisierung besitzt. Erst eine Pathologie, wie z. B. die proximale Skaphoidpseudarthrose oder die dislozierte proximale Skaphoidfraktur können dann $\mathrm{zu}$ entsprechenden Durchblutungsstörungen führen. Allein die anatomische Beziehung des Skaphoids zu den intrinsischen und extrinsischen Bandsystemen im Bereich der karpalen Knochen sind eigene wochenfüllende Themen.

Die erste Arbeit bringt einleitend die Anatomie und Biomechanik des Skaphoids auf den neusten Stand.

\section{》) Die Vitalitätsbeurteilung ist entscheidend für das Operationsverfahren}

Der Schwerpunkt der radiologischen Arbeit liegt in der Darstellung der heutigen Möglichkeiten der präoperativen Vitalitätsbeurteilung (Perfusionsdarstellung). Diese Vitalitätsbeurteilung ist der wesentliche Baustein der Indikationsfin- dung für die Wahl des Operationsverfahrens.

In einer zusätzlichen Arbeit wird die aktuell entwickelte S3-Leitlinie für Skaphoidfrakturen in ihren wesentlichen Bestandteilen skizziert.

Die Indikation zur operativen Verwendung von avaskulären Beckenkammund Radiustransplantaten bei dem Vorliegen einer Skaphoidpseudarthrose wird zunehmend kontrovers diskutiert. In dieser Arbeit wird u. a. genau analysiert, welche Klassifikationen zur Beurteilung hilfreich sind.

Des Weiteren werden in zwei Arbeiten die neueren und älteren Techniken der gefäßgestielten und freien gefäßgestielten Knochentransplantate als mögliche Therapieoptionen der Skaphoidpseudarthrose aufgezeigt (Zaidembergtransplantat/Femurtransplantat).

Am Schluss wird das Skaphoid aus der Sicht des rheumatischen Formenkreises durch eine entsprechende Originalarbeit beleuchtet.

Durch die Weiterentwicklung von gefäßgestielten und freien gefäßgestielten Transplantaten unter Berücksichtigung der ebenfalls sehr guten Therapie durch avaskuläre Transplantate ist nun hier erneut akuter Handlungsbedarf gegeben, um eine adäquate Systematisierung hinsichtlich der Indikationsstellung zu den jeweiligen Verfahren schaffen zu können. Die Indikationsstellung sowie die hierfür notwendige Diagnostik sowie klare Definition mit entsprechenden Klassifikationen ist neu entbrannt.

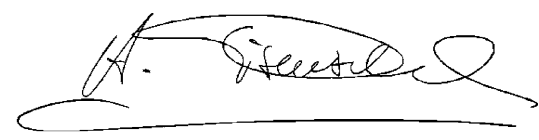

Prof. Dr. A. Eisenschenk

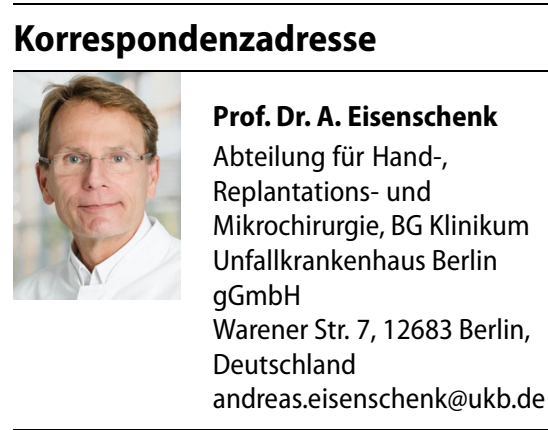

Interessenkonflikt. A. Eisenschenk gibt an, dass kein Interessenkonflikt besteht. 